\title{
The Generalized Poynting Theorem for the General Field and Solution of the 4/3 Problem
}

\author{
Sergey G. Fedosin \\ PO box 614088, Sviazeva str. 22-79, Perm, Perm Krai, Russia \\ fedosin@hotmail.com
}

Keywords: Poynting theorem; acceleration field; pressure field; 4/3 problem; system's energy.

\begin{abstract}
The generalized Poynting theorem is applied to the equilibrium system of particles, both inside and outside the system. The particles are bound to each other by means of the electromagnetic and gravitational fields, acceleration field and pressure field. As a result, the correlation is found between the acceleration field coefficient, the pressure field coefficient, the gravitational constant and the vacuum permittivity. This correlation also depends on the ratio of the charge density to the mass density of the particles inside the sphere. Due to the correlation between the given field coefficients the $4 / 3$ problem is solved and the expression for the relativistic energy of the system is refined.
\end{abstract}

\section{Introduction}

The Poynting theorem is most widely used in electrodynamics, which describes the relation between the rate of change of the energy density of the electromagnetic field inside a certain volume and the electromagnetic energy flux through the surface of this volume, as well as the capacity of work done by the field to move the charges calculated per volume unit [1]. In the full physical system all fields acting in it are taken into account and the stress-energy tensor of matter is introduced into consideration. As a result, the generalized Poynting theorem (or the Umov-Poynting theorem) becomes valid. In particular, the work done by the fields to move the masses and charges per volume unit is equated to the change in the energy density and energy flux in the stress-energy tensor of matter. This leads to the fact that the Poynting theorem for the full physical system in an arbitrary volume can be expressed only in terms of the rate of change of the energy density of all the fields and the energy of matter in this volume, as well as in terms of the energy fluxes of the fields and the energy of matter through the surface surrounding this volume [2]. Thus, the generalized Poynting theorem is transformed into the energy balance equation for the fields and matter.

In the general field concept [3], all the macroscopic particular fields can be included in one of the two components, depending on which four-current is their source [4]. The mass four-current $J^{\mu}$ generates such vector fields, as the gravitational field, acceleration field, pressure field, dissipation field, macroscopic fields of strong and weak interactions. All these fields are included in the mass component of the general field. The charge component of the general field is represented by the electromagnetic field and its source is the charge four-current $j^{\mu}$.

In contrast to the standard approach, in the general field concept the stress-energy tensor of matter is not used at all. The stress-energy tensor of the acceleration field is used instead [5,6]. This means that the description of the kinetic matter energy and the flux of this energy ceases to be the phenomenological description, requiring adjustment to the results of experiments, but is carried out as in a covariant way using the terms of the velocity field and the corresponding acceleration field.

The concept of the acceleration field is intended for a more accurate description of the accelerated motion of particles in a physical system. In the first approximation, we can assume that the typical particles of the system behave like non-rotating solid bodies during interaction. This corresponds to the fact that only the scalar potential of the acceleration field is used, and the proper vector potential of each particle is not taken into account. However, even in case of parallel transport of a solid particle along a curved line, we can see that where the instantaneous radius of 
rotation is larger, the matter moves at a higher velocity than the velocity of the particle's center of mass. In case of the particle's rotation, its energy would differ from the rest energy of a stationary and non-rotating particle. The analysis of such cases is significantly simplified when taking into account the vector potential of the acceleration field as a new degree of freedom in the description of motion. Since the scalar and vector potentials form the four-potential, the acceleration field becomes a vector field. The four-potential of the acceleration field of a rectilinearly moving solid particle coincides with its four-velocity. However, in the general case, the four-potential is found by solving the wave equation of the acceleration field in a way similar to that for other vector fields.

The same situation takes place for the pressure field. As a rule, the pressure field is considered either as a purely scalar field or as a certain effective vector field, in which the proper vector potential of the pressure field of a typical particle is always equal to zero. In the latter case, the role of the four-potential of the pressure field in the system's Lagrangian is played by the product of the pressure by the particle's four-velocity, divided by the square of the speed of light. Instead, we consider an actual vector pressure field, when its four-potential is determined by means of the corresponding four-dimensional wave equation. This means that for the continuously distributed matter and a large number of interacting particles, the global four-potentials of the acceleration field and the pressure field are determined at the same time by all the particles of the system and no longer depend on the four-velocity of the particle under consideration.

Next, we will analyze the generalized Poynting theorem for both components of the general field. This will lead us to the relations that link the coefficients of the general field, acceleration field and pressure field. In addition, we will provide the solution of $4 / 3$ problem and a more precise expression for the relativistic energy of the system.

Note that we consider the gravitational field as a vector field in the framework of the covariant theory of gravitation, similar to the approach developed in [7].

In order to obtain exact results without loss of generality, in analyzing the generalized Poynting theorem we will rely on the relativistic uniform model [5]. This model describes a stable physical system, the particles of which are in equilibrium motion under the action of the global fields of the system. In such a system, we can quite easily calculate the potentials and field strengths, find the field tensors and the corresponding stress-energy tensors, which are necessary for verifying the generalized Poynting theorem and solving the $4 / 3$ problem.

\section{The Covariant Form of the Generalized Poynting Theorem}

The equation of motion of matter in two-component general field is written as follows [4]:

$$
F_{\mu \nu} j^{v}+s_{\mu \nu} J^{v}=-\nabla^{v}\left(W_{\mu \nu}+T_{\mu \nu}\right)=-\nabla^{v} \tau_{\mu \nu}=0,
$$

where $F_{\mu \nu}$ is the electromagnetic tensor, $s_{\mu \nu}$ is the tensor of the mass component of the general field, $W_{\mu \nu}$ is the stress-energy tensor of the electromagnetic field, $T_{\mu \nu}$ is the stress-energy tensor of the mass component of the general field, $\tau_{\mu \nu}$ is the total stress-energy tensor of the physical system of matter and all the fields acting in the system.

The generalized Poynting theorem follows from (1), provided that in the equation of motion the index value $\mu=0$ is selected:

$$
F_{0 v} j^{v}+s_{0 v} J^{v}=-\nabla^{v}\left(W_{0 v}+T_{0 v}\right)=-\nabla^{v} \tau_{0 v}=0
$$

Tensor-differential equation (2) describes the rate of change of the total energy of fields in an arbitrary volume, depending on the fluxes of these fields directed inside this volume. If in (1) we 
select the index $\mu=1,2,3$, this leads to the equation of motion of matter in the general field in the curved spacetime. As it is shown in [4], the left side of the equation of motion will contain the product of the mass density by the four-acceleration and the right side will contain the sum of densities of the four-forces from the fields acting on the matter.

In the standard approach, the expression $-\nabla^{v} \tau_{0 v}=-\nabla_{v} \tau^{0 v}=0$ is used for the generalized Poynting theorem, while the tensor $\tau_{\mu \nu}$ includes the stress-energy tensor of matter. In the general theory of relativity the tensor $\tau_{\mu \nu}$ takes on an additional meaning, because in this theory not only the equation $\nabla^{v} \tau_{\mu \nu}=0$ is considered as an equation of motion, but also the equation for the metric, containing the tensor $\tau_{\mu \nu}$, determines the metric, which is equivalent to the gravitational field in a tensor form [8].

The problem of this approach is the fact that the form of the stress-energy tensor of matter is not commonly-accepted and uniquely determined, and it depends on whether it includes the contributions of pressure, internal matter energy and other factors that are not clearly defined. The form of this tensor also depends considerably on the form (scalar, vector, tensor), in which these factors are taken into account in the Lagrangian.

In our approach, we avoid such uncertainty, because instead of the stress-energy tensor of matter we use the uniquely determined stress-energy tensor of the acceleration field, and the contribution of pressure is taken into account with the help of a separate, covariantly determined stress-energy tensor of the pressure field. The contributions from the dissipation field [9], as well as other fields can be taken into account in a similar way. It was thanks to the acceleration field and the vector pressure field in [9] that for the first time it was possible to accurately reproduce in the curved spacetime the phenomenological Navier-Stokes equation in a covariant way.

The tensor $s_{\mu \nu}$ of the mass component of the general field is expressed in terms of the tensors of particular fields. For brevity, we will further limit ourselves to the electromagnetic field and three more basic fields, such as the gravitational field, acceleration field and pressure field. The corresponding sum of tensors of these three fields gives the tensor $s_{\mu \nu}$ :

$$
s_{\mu v}=\Phi_{\mu v}+u_{\mu v}+f_{\mu v} .
$$

Substituting (3) into (1) yields the following:

$$
F_{\mu \nu} j^{v}+\left(\Phi_{\mu v}+u_{\mu \nu}+f_{\mu \nu}\right) J^{v}=-\nabla^{v}\left(W_{\mu v}+T_{\mu \nu}\right)=0
$$

while the stress-energy tensor $T_{\mu \nu}$ of the mass component of the general field depends on the tensor $s_{\mu \nu}$ and the general field coefficient $\varpi$ :

$$
T_{\mu \nu}=\frac{c^{2}}{4 \pi \varpi}\left(-g_{\beta \nu} s_{\kappa \mu} s^{\kappa \beta}+\frac{1}{4} g_{\mu \nu} s_{\alpha \beta} s^{\alpha \beta}\right)=\frac{c^{2}}{4 \pi \varpi} g^{\alpha \kappa}\left(-\delta_{\mu}^{\beta} \delta_{v}^{\sigma}+\frac{1}{4} g_{\mu \nu} g^{\sigma \beta}\right) s_{\alpha \beta} s_{\kappa \sigma} .
$$

In turn, the tensor of the mass component of the general field $s_{\mu v}$ can be found from the field equations:

$$
\nabla_{\beta} s^{\alpha \beta}=-\frac{4 \pi \varpi}{c^{2}} J^{\alpha}, \quad \quad \varepsilon^{\alpha \beta \gamma \delta} \nabla_{\gamma} s_{\alpha \beta}=0
$$


The stress-energy tensor $W_{\mu \nu}$ and the electromagnetic field equations have the standard form:

$$
\begin{array}{cc}
W_{\mu \nu}=\varepsilon_{0} c^{2}\left(-g_{\beta \nu} F_{\kappa \mu} F^{\kappa \beta}+\frac{1}{4} g_{\mu \nu} F_{\alpha \beta} F^{\alpha \beta}\right)=\varepsilon_{0} c^{2} g^{\alpha \kappa}\left(-\delta_{\mu}^{\beta} \delta_{v}^{\sigma}+\frac{1}{4} g_{\mu \nu} g^{\sigma \beta}\right) F_{\alpha \beta} F_{\kappa \sigma} . \\
\nabla_{\beta} F^{\alpha \beta}=-\frac{1}{c^{2} \varepsilon_{0}} j^{\alpha}, & \varepsilon^{\alpha \beta \gamma \delta} \nabla_{\gamma} F_{\alpha \beta}=0 .
\end{array}
$$

In these expressions: $\varepsilon_{0}$ is the vacuum permittivity, $c$ is the speed of light, $g_{\mu v}$ is the metric tensor, $\delta_{\mu}^{\beta}$ is the Kronecker symbol, $\varepsilon^{\alpha \beta \gamma \delta}$ is the Levi-Civita symbol.

Equation (4) can be analyzed by two methods. In the first of them, we solve equations (6) and (8), find the tensors $s_{\mu \nu}$ and $F_{\mu \nu}$ and then the tensors $T_{\mu v}$ and $W_{\mu v}$ in (5) and (7), which then we substitute into (4) to calculate the divergence of the sum of these tensors. The second method does not require finding the tensors $T_{\mu \nu}$ and $W_{\mu \nu}$, in this case we use the left-hand side of (4).

The stress-energy tensor of the gravitational field according to [10] and the stress-energy tensors of the acceleration field and pressure field according to [5] have the following form:

$$
\begin{gathered}
U_{\mu \nu}=-\frac{c^{2}}{4 \pi G}\left(-g_{\beta \nu} \Phi_{\kappa \mu} \Phi^{\kappa \beta}+\frac{1}{4} g_{\mu \nu} \Phi_{\alpha \beta} \Phi^{\alpha \beta}\right)=-\frac{c^{2}}{4 \pi G} g^{\alpha \kappa}\left(-\delta_{\mu}^{\beta} \delta_{v}^{\sigma}+\frac{1}{4} g_{\mu \nu} g^{\sigma \beta}\right) \Phi_{\alpha \beta} \Phi_{\kappa \sigma} . \\
B_{\mu \nu}=\frac{c^{2}}{4 \pi \eta}\left(-g_{\beta \nu} u_{\kappa \mu} u^{\kappa \beta}+\frac{1}{4} g_{\mu \nu} u_{\alpha \beta} u^{\alpha \beta}\right)=\frac{c^{2}}{4 \pi \eta} g^{\alpha \kappa}\left(-\delta_{\mu}^{\beta} \delta_{v}^{\sigma}+\frac{1}{4} g_{\mu \nu} g^{\sigma \beta}\right) u_{\alpha \beta} u_{\kappa \sigma} . \\
P_{\mu \nu}=\frac{c^{2}}{4 \pi \sigma}\left(-g_{\beta \nu} f_{\kappa \mu} f^{\kappa \beta}+\frac{1}{4} g_{\mu \nu} f_{\alpha \beta} f^{\alpha \beta}\right)=\frac{c^{2}}{4 \pi \sigma} g^{\alpha \kappa}\left(-\delta_{\mu}^{\beta} \delta_{v}^{\sigma}+\frac{1}{4} g_{\mu \nu} g^{\sigma \beta}\right) f_{\alpha \beta} f_{\kappa \sigma} .
\end{gathered}
$$

If we assume that all these fields exist independently of each other and their contribution into the Lagrangian is made independently, then the following wave equations hold true for the tensors of these fields:

$$
\begin{array}{ll}
\nabla_{\beta} \Phi^{\alpha \beta}=\frac{4 \pi G}{c^{2}} J^{\alpha}, & \varepsilon^{\alpha \beta \gamma \delta} \nabla_{\gamma} \Phi_{\alpha \beta}=0 . \\
\nabla_{\beta} u^{\alpha \beta}=-\frac{4 \pi \eta}{c^{2}} J^{\alpha}, & \varepsilon^{\alpha \beta \delta} \nabla_{\gamma} u_{\alpha \beta}=0 . \\
\nabla_{\beta} f^{\alpha \beta}=-\frac{4 \pi \sigma}{c^{2}} J^{\alpha}, & \varepsilon^{\alpha \beta \gamma \delta} \nabla_{\gamma} f_{\alpha \beta}=0 .
\end{array}
$$

In (9-14) $G$ is the gravitational constant, $\eta$ is the acceleration field coefficient, $\sigma$ is the pressure field coefficient.

Summing up the first equations in (12-14), which contain the divergences of the field tensors, taking into account (3) and comparing the result with the divergence of the mass component of the general field $s^{\alpha \beta}$ in (6), we arrive at the following:

$$
\nabla_{\beta}\left(\Phi^{\alpha \beta}+u^{\alpha \beta}+f^{\alpha \beta}\right)=\nabla_{\beta} s^{\alpha \beta}=\frac{4 \pi(G-\eta-\sigma)}{c^{2}} J^{\alpha}=-\frac{4 \pi \varpi}{c^{2}} J^{\alpha},
$$




$$
\varpi=\eta+\sigma-G
$$

We will now transform the composition of tensors $s_{\alpha \beta} s_{\kappa \sigma}$ with the help of (3):

$$
\begin{aligned}
& s_{\alpha \beta} s_{\kappa \sigma}=\left(\Phi_{\alpha \beta}+u_{\alpha \beta}+f_{\alpha \beta}\right)\left(\Phi_{\kappa \sigma}+u_{\kappa \sigma}+f_{\kappa \sigma}\right)=\Phi_{\alpha \beta} \Phi_{\kappa \sigma}+u_{\alpha \beta} u_{\kappa \sigma}+f_{\alpha \beta} f_{\kappa \sigma}+ \\
& +\Phi_{\alpha \beta}\left(u_{\kappa \sigma}+f_{\kappa \sigma}\right)+u_{\alpha \beta}\left(\Phi_{\kappa \sigma}+f_{\kappa \sigma}\right)+f_{\alpha \beta}\left(\Phi_{\kappa \sigma}+U_{\kappa \sigma}\right) .
\end{aligned}
$$

We can substitute this equation into (5) and then take into account the expression for the tensors (9-11):

$$
\begin{aligned}
& T_{\mu \nu}=-\frac{G}{\varpi} U_{\mu \nu}+\frac{\eta}{\varpi} B_{\mu \nu}+\frac{\sigma}{\varpi} P_{\mu \nu}+ \\
& +\frac{c^{2}}{4 \pi \varpi} g^{\alpha \kappa}\left(-\delta_{\mu}^{\beta} \delta_{v}^{\sigma}+\frac{1}{4} g_{\mu \nu} g^{\sigma \beta}\right)\left[\Phi_{\alpha \beta}\left(u_{\kappa \sigma}+f_{\kappa \sigma}\right)+u_{\alpha \beta}\left(\Phi_{\kappa \sigma}+f_{\kappa \sigma}\right)+f_{\alpha \beta}\left(\Phi_{\kappa \sigma}+u_{\kappa \sigma}\right)\right] .
\end{aligned}
$$

Field equations (12-14) were solved in $[11,12]$ for a massive charged spherical body, wherein it was found that field tensors have similar components. In particular, we can write the following:

$$
\frac{\Phi_{\kappa \sigma}}{G}=-\frac{4 \pi \varepsilon_{0} \rho_{0} F_{\kappa \sigma}}{\rho_{0 q}}=-\frac{u_{\kappa \sigma}}{\eta}=-\frac{f_{\kappa \sigma}}{\sigma} .
$$

Substituting (17) into (16), in view of (15) we find:

$$
T_{\mu \nu}=\frac{\eta+\sigma-G}{\varpi}\left(U_{\mu \nu}+B_{\mu \nu}+P_{\mu \nu}\right)=U_{\mu \nu}+B_{\mu \nu}+P_{\mu \nu}
$$

Thus, if we consider all the fields independent of each other, then inside the body the stressenergy tensor of the mass component of the general field is equal to the sum of the stress-energy tensors of all the fields, except for the electromagnetic field, and the general field coefficient $\varpi$ is expressed in terms of the coefficients of the other fields, according to (15).

\section{The Generalized Poynting Theorem in the Weak Field Limit}

\subsection{The analysis of equations inside the body}

Let us substitute (18) into the right side of (4) with the index $\mu=0$ :

$$
-\nabla^{v}\left(W_{0 v}+U_{0 v}+B_{0 v}+P_{0 v}\right)=0
$$

We will consider equation (19) in the weak field limit, when the covariant derivative is transformed into the four-gradient and the curvature of spacetime can be neglected. This case corresponds to the Minkowski space of the special theory of relativity. From (19) it follows:

$$
\frac{\partial\left(W_{00}+U_{00}+B_{00}+P_{00}\right)}{\partial t}+\nabla \cdot(\mathbf{P}+\mathbf{H}+\mathbf{K}+\mathbf{F})=0 .
$$

In (20) the Poynting vector of the electromagnetic field, the Heaviside vector of the gravitational field, the vectors of the energy flux density of the acceleration field and pressure fields, respectively, are defined by the following formulas: 


$$
\mathbf{P}=\varepsilon_{0} c^{2}[\mathbf{E} \times \mathbf{B}], \quad \mathbf{H}=-\frac{c^{2}}{4 \pi G}[\mathbf{\Gamma} \times \mathbf{\Omega}], \quad \mathbf{K}=\frac{c^{2}}{4 \pi \eta}[\mathbf{S} \times \mathbf{N}], \quad \mathbf{F}=\frac{c^{2}}{4 \pi \sigma}[\mathbf{C} \times \mathbf{I}]
$$

Energy densities of the fields have the form:

$$
\begin{array}{lr}
W_{00}=\frac{\varepsilon_{0}}{2}\left[E^{2}+c^{2} B^{2}\right], & U_{00}=-\frac{1}{8 \pi G}\left[\Gamma^{2}+c^{2} \Omega^{2}\right] \\
B_{00}=\frac{1}{8 \pi \eta}\left[S^{2}+c^{2} N^{2}\right], & P_{00}=\frac{1}{8 \pi \sigma}\left[C^{2}+c^{2} I^{2}\right] .
\end{array}
$$

In (21-22) the vectors of the energy flux density and the energy densities are expressed in terms of the corresponding field strengths and solenoidal vectors of the field.

Suppose there is a body of spherical shape, which is in equilibrium under the action of its own gravitational and electromagnetic fields, acceleration field and pressure field. This body in general is not rotating and is motionless relative to the reference frame $K^{\prime}$, while the center of the coordinate system is located in the center of the sphere. The body under consideration consists of a number of small randomly moving particles with the mass density $\rho_{0}$ and charge density $\rho_{0 q}$ in the reference frames associated with the particles. If the space between the particles is small, as in liquids, we can assume that the matter inside the body is distributed continuously. We studied such a physical system in $[12,13]$. Due to the randomness of particles' motion, in $K^{\prime}$ the vector field potentials and, accordingly, the solenoidal vectors: B (magnetic induction), $\boldsymbol{\Omega}$ (torsion field), $\mathbf{N}$ and $\mathbf{I}$ are equal to zero.

In addition, we found expressions for the electric field strength $\mathbf{E}$, gravitational field strength $\boldsymbol{\Gamma}$, and acceleration field strength $\mathbf{S}$ and pressure field strength $\mathbf{C}$ inside the body as a function of the current radius. After that, in [12] we made Lorentz transformation of coordinates from the reference frame $K^{\prime}$ into the laboratory reference frame $K_{L}$, relative to which the body is moving at the velocity $v$ along the axis $O X$.

The vectors $\mathbf{E}$ and $\mathbf{B}$ are part of the components of the electromagnetic tensor $F_{\mu \nu}$, therefore in Lorentz transformations they are transformed in a special way - as tensor components. It is enough to know the vectors $\mathbf{E}$ and $\mathbf{B}$ in $K^{\prime}$ in order to find their form in the laboratory reference frame $K_{L}$.

Another way to find the vectors $\mathbf{E}$ and $\mathbf{B}$ in $K_{L}$ is as follows: first we need to transform the four-potential of the electromagnetic field $A_{\mu}$ from $K^{\prime}$ into $K_{L}$ with the help of Lorentz transformations, and then to calculate the electromagnetic tensor components in $K_{L}$ by the formula: $F_{\mu \nu}=\partial_{\mu} A_{v}-\partial_{\nu} A_{\mu}$. In both cases, for the electromagnetic field inside the moving sphere, after simplifying the expression for the scalar field potential, we obtain the following:

$$
\begin{array}{cl}
E_{x}=\frac{\gamma \gamma_{c} \rho_{0 q}(x-v t)}{3 \varepsilon_{0}}, \quad E_{y}=\frac{\gamma \gamma_{c} \rho_{0 q} y}{3 \varepsilon_{0}}, & E_{z}=\frac{\gamma \gamma_{c} \rho_{0 q} z}{3 \varepsilon_{0}}, \\
B_{x}=0, & B_{y}=-\frac{\gamma \gamma_{c} \rho_{0 q} z v}{3 \varepsilon_{0} c^{2}},
\end{array}
$$


In (23) the Lorentz factor $\gamma=\frac{1}{\sqrt{1-v^{2} / c^{2}}}$ arises from the motion of the sphere at the constant velocity $\mathbf{v}$ in the reference frame $K_{L}$, and $\gamma_{c}=\frac{1}{\sqrt{1-v_{c}^{2} / c^{2}}}$ is the Lorentz factor for the velocities $v_{c}$ of the particles in the center of the sphere in the reference frame $K^{\prime}$. At the time point $t=0$ the sphere's center is located at the origin of the reference frame $K_{L}$.

The gravitational field strength $\boldsymbol{\Gamma}$ and the torsion field $\mathbf{\Omega}$ inside the moving sphere under the same conditions will be as follows:

$$
\begin{gathered}
\Gamma_{x}=-\frac{4 \pi G \gamma \gamma_{c} \rho_{0}(x-v t)}{3}, \quad \Gamma_{y}=-\frac{4 \pi G \gamma \gamma_{c} \rho_{0} y}{3}, \quad \Gamma_{z}=-\frac{4 \pi G \gamma \gamma_{c} \rho_{0} z}{3}, \\
\Omega_{x}=0, \quad \Omega_{y}=\frac{4 \pi G \gamma \gamma_{c} \rho_{0} z v}{3 c^{2}}, \quad \Omega_{z}=-\frac{4 \pi G \gamma \gamma_{c} \rho_{0} y v}{3 c^{2}} .
\end{gathered}
$$

The components of the acceleration field and the pressure field have similar expressions:

$$
\begin{gathered}
S_{x}=\frac{4 \pi \eta \gamma \gamma_{c} \rho_{0}(x-v t)}{3}, \quad S_{y}=\frac{4 \pi \eta \gamma \gamma_{c} \rho_{0} y}{3}, \quad S_{z}=\frac{4 \pi \eta \gamma \gamma_{c} \rho_{0} z}{3}, \\
N_{x}=0, \quad N_{y}=-\frac{4 \pi \eta \gamma \gamma_{c} \rho_{0} z v}{3 c^{2}}, \quad N_{z}=\frac{4 \pi \eta \gamma \gamma_{c} \rho_{0} y v}{3 c^{2}} . \\
C_{x}=\frac{4 \pi \sigma \gamma \gamma_{c} \rho_{0}(x-v t)}{3}, \quad C_{y}=\frac{4 \pi \sigma \gamma \gamma_{c} \rho_{0} y}{3}, \quad C_{z}=\frac{4 \pi \sigma \gamma \gamma_{c} \rho_{0} z}{3}, \\
I_{x}=0, \quad I_{y}=-\frac{4 \pi \sigma \gamma \gamma_{c} \rho_{0} z v}{3 c^{2}}, \quad I_{z}=\frac{4 \pi \sigma \gamma \gamma_{c} \rho_{0} y v}{3 c^{2}} .
\end{gathered}
$$

As we can see, in the physical system under consideration the field strengths and their solenoidal vectors in (24-26), included in the tensor components $\Phi_{\alpha \beta}, u_{\alpha \beta}$ and $f_{\alpha \beta}$, satisfy relation (17).

With the help of (23-26) we can calculate the components of the field energy flux vectors (21) and their divergences. For the electromagnetic field:

$$
\begin{array}{ll}
P_{x}=\frac{\gamma^{2} \gamma_{c}^{2} \rho_{0 q}^{2}\left(y^{2}+z^{2}\right) v}{9 \varepsilon_{0}}, & P_{y}=-\frac{\gamma^{2} \gamma_{c}^{2} \rho_{0 q}^{2} y(x-v t) v}{9 \varepsilon_{0}}, \\
P_{z}=-\frac{\gamma^{2} \gamma_{c}^{2} \rho_{0 q}^{2} z(x-v t) v}{9 \varepsilon_{0}}, & \nabla \cdot \mathbf{P}=-\frac{2 \gamma^{2} \gamma_{c}^{2} \rho_{0 q}^{2}(x-v t) v}{9 \varepsilon_{0}} .
\end{array}
$$

For the gravitational field:

$$
H_{x}=-\frac{4 \pi G \gamma^{2} \gamma_{c}^{2} \rho_{0}^{2}\left(y^{2}+z^{2}\right) v}{9}, \quad H_{y}=\frac{4 \pi G \gamma^{2} \gamma_{c}^{2} \rho_{0}^{2} y(x-v t) v}{9}
$$




$$
H_{z}=\frac{4 \pi G \gamma^{2} \gamma_{c}^{2} \rho_{0}^{2} z(x-v t) v}{9}, \quad \nabla \cdot \mathbf{H}=\frac{8 \pi G \gamma^{2} \gamma_{c}^{2} \rho_{0}^{2}(x-v t) v}{9}
$$

For the acceleration field:

$$
\begin{array}{cc}
K_{x}=\frac{4 \pi \eta \gamma^{2} \gamma_{c}^{2} \rho_{0}^{2}\left(y^{2}+z^{2}\right) v}{9}, & K_{y}=-\frac{4 \pi \eta \gamma^{2} \gamma_{c}^{2} \rho_{0}^{2} y(x-v t) v}{9}, \\
K_{z}=-\frac{4 \pi \eta \gamma^{2} \gamma_{c}^{2} \rho_{0}^{2} z(x-v t) v}{9}, & \nabla \cdot \mathbf{K}=-\frac{8 \pi \eta \gamma^{2} \gamma_{c}^{2} \rho_{0}^{2}(x-v t) v}{9} .
\end{array}
$$

For the pressure field:

$$
\begin{gathered}
F_{x}=\frac{4 \pi \sigma \gamma^{2} \gamma_{c}^{2} \rho_{0}^{2}\left(y^{2}+z^{2}\right) v}{9}, \quad F_{y}=-\frac{4 \pi \sigma \gamma^{2} \gamma_{c}^{2} \rho_{0}^{2} y(x-v t) v}{9} \\
F_{z}=-\frac{4 \pi \sigma \gamma^{2} \gamma_{c}^{2} \rho_{0}^{2} z(x-v t) v}{9}, \quad \nabla \cdot \mathbf{F}=-\frac{8 \pi \sigma \gamma^{2} \gamma_{c}^{2} \rho_{0}^{2}(x-v t) v}{9} .
\end{gathered}
$$

Expression (28) for the components of the energy flux vector of the gravitational field inside the sphere is the same as in [14], where the gravitational field momentum is calculated. sphere:

With the help of (23-26) we find the energy densities of the fields (22) inside the moving

$$
\begin{array}{r}
W_{00}=\frac{\gamma^{2} \gamma_{c}^{2} \rho_{0 q}^{2}}{18 \varepsilon_{0}}\left[(x-v t)^{2}+\left(y^{2}+z^{2}\right)\left(1+\frac{v^{2}}{c^{2}}\right)\right] . \\
U_{00}=-\frac{2 \pi G \gamma^{2} \gamma_{c}^{2} \rho_{0}^{2}}{9}\left[(x-v t)^{2}+\left(y^{2}+z^{2}\right)\left(1+\frac{v^{2}}{c^{2}}\right)\right] . \\
B_{00}=\frac{2 \pi \eta \gamma^{2} \gamma_{c}^{2} \rho_{0}^{2}}{9}\left[(x-v t)^{2}+\left(y^{2}+z^{2}\right)\left(1+\frac{v^{2}}{c^{2}}\right)\right] . \\
P_{00}=\frac{2 \pi \sigma \gamma^{2} \gamma_{c}^{2} \rho_{0}^{2}}{9}\left[(x-v t)^{2}+\left(y^{2}+z^{2}\right)\left(1+\frac{v^{2}}{c^{2}}\right)\right] .
\end{array}
$$

Let us substitute (27-31) into expression (20) for the Poynting theorem, taking into account all the four existing fields. This gives the following:

$$
\eta+\sigma=G-\frac{\rho_{0 q}^{2}}{4 \pi \varepsilon_{0} \rho_{0}^{2}} .
$$

In (32) we found a relationship between the acceleration field coefficient $\eta$, the pressure field coefficient $\sigma$, the gravitational constant $G$ and the vacuum permittivity $\varepsilon_{0}$. This relationship also depends on the ratio of the charge density $\rho_{0 q}$ to the mass density $\rho_{0}$ of the particles inside the sphere, measured in the reference frames associated with these particles. It is assumed that the ratio 
$\frac{\rho_{0 q}}{\rho_{0}}$ is a constant. An ideal case is possible, when the particles inside the sphere are not charged and the entire electric charge is located in the sphere's shell. Then for the particles inside the sphere $\rho_{0 q}=0$, and the contribution from the electromagnetic field in (32) vanishes. If we substitute (32) into (15), then the coefficient of the general field inside the sphere depends only on the electromagnetic component:

$$
\varpi=-\frac{\rho_{0 q}^{2}}{4 \pi \varepsilon_{0} \rho_{0}^{2}} .
$$

In case, when the matter inside the sphere is neutral and $\rho_{0 q}=0$, the coefficient $\varpi$ tends to zero.

Relation (32) is a manifestation of the fact that at each point of the physical system under consideration the sum of the energy densities of all the fields inside the body, included in the time components of the tensors in (20), is equal to zero. The sum of the energy flux vectors of all the fields inside the body is also equal to zero.

\subsection{The analysis of equations outside the body}

Let us now pass on to the fields outside the body, where there is no matter and only the electromagnetic and gravitational fields are left. In the reference frame $K^{\prime}$ the strengths of both fields look as if the entire charge and mass are concentrated at the center of the fixed sphere. This is due to the fact that the field strengths are inversely proportional to the square of the distance from the sphere's center to the point where the strengths are determined. The electromagnetic field strength turns out to be directly proportional to the total charge $q$ of the sphere filled with particles, and the gravitational field strength is proportional to the mass $m$ of the sphere. With the help of Lorentz transformations we can turn from the reference frame $K^{\prime}$ to the laboratory reference frame $K_{L}$, in which the sphere with the particles is moving at the velocity $v$ along the axis $O X$. Conversion of the components of the electromagnetic and gravitational fields into the reference frame $K_{L}$ has been done in [12]. Outside the moving sphere these components are as follows:

$$
\begin{gathered}
E_{x}=\frac{\gamma \gamma_{c} q(x-v t)}{4 \pi \varepsilon_{0}\left[\gamma^{2}(x-v t)^{2}+y^{2}+z^{2}\right]^{3 / 2}}, \quad E_{y}=\frac{\gamma \gamma_{c} q y}{4 \pi \varepsilon_{0}\left[\gamma^{2}(x-v t)^{2}+y^{2}+z^{2}\right]^{3 / 2}}, \\
E_{z}=\frac{\gamma \gamma_{c} q z}{4 \pi \varepsilon_{0}\left[\gamma^{2}(x-v t)^{2}+y^{2}+z^{2}\right]^{3 / 2}}, \\
B_{y}=-\frac{B_{x}=0,}{4 \pi \varepsilon_{0} c^{2}\left[\gamma^{2}(x-v t)^{2}+y^{2}+z^{2}\right]^{3 / 2}}, \quad B_{z}=\frac{\gamma \gamma_{c} q y v}{4 \pi \varepsilon_{0} c^{2}\left[\gamma^{2}(x-v t)^{2}+y^{2}+z^{2}\right]^{3 / 2}} . \\
\Gamma_{x}=-\frac{G \gamma \gamma_{c} m(x-v t)}{\left[\gamma^{2}(x-v t)^{2}+y^{2}+z^{2}\right]^{3 / 2}}, \quad \Gamma_{y}=-\frac{G \gamma \gamma_{c} m y}{\left[\gamma^{2}(x-v t)^{2}+y^{2}+z^{2}\right]^{3 / 2}}, \\
\Gamma_{z}=-\frac{G \gamma \gamma_{c} m z}{\left[\gamma^{2}(x-v t)^{2}+y^{2}+z^{2}\right]^{3 / 2}},
\end{gathered}
$$




$$
\Omega_{y}=\frac{G \gamma \gamma_{c} m z v}{c^{2}\left[\gamma^{2}(x-v t)^{2}+y^{2}+z^{2}\right]^{3 / 2}}, \quad \Omega_{z}=-\frac{G \gamma \gamma_{c} m y v}{c^{2}\left[\gamma^{2}(x-v t)^{2}+y^{2}+z^{2}\right]^{3 / 2}} .
$$

Using (34-35), we calculate the components of the field energy flux vectors (21) and their divergences. For the electromagnetic field:

$$
\begin{gathered}
P_{x}=\frac{\gamma^{2} \gamma_{c}^{2} q^{2}\left(y^{2}+z^{2}\right) v}{16 \pi^{2} \varepsilon_{0}\left[\gamma^{2}(x-v t)^{2}+y^{2}+z^{2}\right]^{3}}, \quad P_{y}=-\frac{\gamma^{2} \gamma_{c}^{2} q^{2} y(x-v t) v}{16 \pi^{2} \varepsilon_{0}\left[\gamma^{2}(x-v t)^{2}+y^{2}+z^{2}\right]^{3}}, \\
P_{z}=-\frac{\gamma^{2} \gamma_{c}^{2} q^{2} z(x-v t) v}{16 \pi^{2} \varepsilon_{0}\left[\gamma^{2}(x-v t)^{2}+y^{2}+z^{2}\right]^{3}}, \\
\nabla \cdot \mathbf{P}=-\frac{\gamma^{2} \gamma_{c}^{2} q^{2}(x-v t)\left[\gamma^{2}(x-v t)^{2}+\left(3 \gamma^{2}-2\right)\left(y^{2}+z^{2}\right)\right] v}{8 \pi^{2} \varepsilon_{0}\left[\gamma^{2}(x-v t)^{2}+y^{2}+z^{2}\right]^{4}} .
\end{gathered}
$$

For the gravitational field:

$$
\begin{gathered}
H_{x}=-\frac{G \gamma^{2} \gamma_{c}^{2} m^{2}\left(y^{2}+z^{2}\right) v}{4 \pi\left[\gamma^{2}(x-v t)^{2}+y^{2}+z^{2}\right]^{3}}, \quad H_{y}=\frac{G \gamma^{2} \gamma_{c}^{2} m^{2} y(x-v t) v}{4 \pi\left[\gamma^{2}(x-v t)^{2}+y^{2}+z^{2}\right]^{3}}, \\
H_{z}=\frac{G \gamma^{2} \gamma_{c}^{2} m^{2} z(x-v t) v}{4 \pi\left[\gamma^{2}(x-v t)^{2}+y^{2}+z^{2}\right]^{3}}, \\
\nabla \cdot \mathbf{H}=\frac{G \gamma^{2} \gamma_{c}^{2} m^{2}(x-v t)\left[\gamma^{2}(x-v t)^{2}+\left(3 \gamma^{2}-2\right)\left(y^{2}+z^{2}\right)\right] v}{2 \pi\left[\gamma^{2}(x-v t)^{2}+y^{2}+z^{2}\right]^{4}}
\end{gathered}
$$

The energy densities of the fields (22) outside the sphere and their partial derivatives with respect to time for the field components (34-35) will be equal to:

$$
\begin{gathered}
W_{00}=\frac{\gamma^{2} \gamma_{c}^{2} q^{2}\left[(x-v t)^{2}+\left(y^{2}+z^{2}\right)\left(1+\frac{v^{2}}{c^{2}}\right)\right]}{32 \pi^{2} \varepsilon_{0}\left[\gamma^{2}(x-v t)^{2}+y^{2}+z^{2}\right]^{3}} . \\
\frac{\partial W_{00}}{\partial t}=\frac{\gamma^{2} \gamma_{c}^{2} q^{2}(x-v t)\left[\gamma^{2}(x-v t)^{2}+\left(3 \gamma^{2}-2\right)\left(y^{2}+z^{2}\right)\right] v}{8 \pi^{2} \varepsilon_{0}\left[\gamma^{2}(x-v t)^{2}+y^{2}+z^{2}\right]^{4}} . \\
U_{00}=-\frac{G \gamma^{2} \gamma_{c}^{2} m^{2}\left[(x-v t)^{2}+\left(y^{2}+z^{2}\right)\left(1+\frac{v^{2}}{c^{2}}\right)\right]}{8 \pi\left[\gamma^{2}(x-v t)^{2}+y^{2}+z^{2}\right]^{3}} .
\end{gathered}
$$




$$
\frac{\partial U_{00}}{\partial t}=-\frac{G \gamma^{2} \gamma_{c}^{2} m^{2}(x-v t)\left[\gamma^{2}(x-v t)^{2}+\left(3 \gamma^{2}-2\right)\left(y^{2}+z^{2}\right)\right] v}{2 \pi\left[\gamma^{2}(x-v t)^{2}+y^{2}+z^{2}\right]^{4}}
$$

The generalized Poynting theorem (20) outside the body can be written as follows:

$$
\frac{\partial\left(W_{00}+U_{00}\right)}{\partial t}+\nabla \cdot(\mathbf{P}+\mathbf{H})=0
$$

After substituting (36-39) in (40) we see that the Poynting theorem outside the body is satisfied separately for each field, while the electromagnetic and gravitational fields do not depend on each other. In each spatial volume variation in time of the energy density of a particular field is exactly compensated by the divergence of the energy flux of this field.

\section{The Equation of Motion of Matter}

Let us consider the left-hand side of equation of motion (4) with respect to the four fields:

$$
F_{\mu \nu} j^{v}+\left(\Phi_{\mu v}+u_{\mu v}+f_{\mu v}\right) J^{v}=0
$$

In the framework of the special theory of relativity, this equation for the spatial tensor components with the index value $\mu=i=1,2,3$ represents the equation of motion of the matter particles and can be expressed in terms of the field strengths and solenoidal vector of the fields:

$$
\frac{\rho_{0 q}}{\rho_{0}} \mathbf{E}+\frac{\rho_{0 q}}{\rho_{0}}[\mathbf{v} \times \mathbf{B}]+\boldsymbol{\Gamma}+[\mathbf{v} \times \mathbf{\Omega}]+\mathbf{S}+[\mathbf{v} \times \mathbf{N}]+\mathbf{C}+[\mathbf{v} \times \mathbf{I}]=0
$$

In the derivation of (41) we used the expressions for the mass four-current $J^{v}=\rho_{0} u^{v}$ and the charge four-current $j^{v}=\rho_{0 q} u^{v}$, as well as the fact that the field tensors consist of the components of field strengths and solenoidal vectors. Let us substitute in (41) the field components from (23-26) inside the moving sphere, given that the vector of the sphere's velocity is directed along the axis $O X$, with the components of the form $\mathbf{v}=(v, 0,0)$. After reducing the nonzero multiplier we obtain the following:

$$
\frac{\rho_{0 q}^{2}}{4 \pi \varepsilon_{0} \rho_{0}^{2}}-G+\eta+\sigma=0
$$

This obtained relation coincides with (32), which was derived for the time components of the tensor equation (4) inside the moving body. This confirms the self-consistency of the found field components with the equations of motion.

\section{Solution of the 4/3 Problem}

Let us calculate the total mass-energy of the four fields inside a spherical body moving at a constant velocity, for which we take integral of the sum of the fields' energy densities over the volume of the moving body and divide it by the speed of light squared and the Lorentz factor. For this we will use expressions (31): 


$$
\begin{aligned}
& m_{f}=\frac{1}{\gamma c^{2}} \int\left(W_{00}+U_{00}+B_{00}+P_{00}\right) d V= \\
& =\frac{2 \pi \gamma \gamma_{c}^{2}}{9 c^{2}} \int \rho_{0}^{2}\left(\frac{\rho_{0 q}^{2}}{4 \pi \varepsilon_{0} \rho_{0}^{2}}-G+\eta+\sigma\right)\left[(x-v t)^{2}+y^{2}+z^{2}+\frac{z^{2} v^{2}}{c^{2}}+\frac{y^{2} v^{2}}{c^{2}}\right] d V=0 .
\end{aligned}
$$

The equality $m_{f}=0$ in (42) follows from expression (32), obtained from the generalized Poynting theorem for the fields inside the body, as well as from the multiplier included in the integral in (42).

Let us now calculate the total mass-energy $m_{p}$ of all the four fields inside the body, moving at a constant velocity, which is associated with the total momentum of these fields. To do this, we need to take the integral over the volume of the moving body of the sum of the fields' momentum densities, while the momentum density of each field is equal to the energy flux density of this field divided by the square of the speed of light. The total momentum of the fields inside the body, obtained by integration, must be equal to the product of the mass-energy $m_{p}$ by the Lorentz factor $\gamma$ and the velocity of the body's motion $\mathbf{v}$ in the reference frame $K_{L}$. For the case when the body's velocity is directed exactly along the axis $O X$ of the reference frame $K_{L}$, we can use the expressions for the components of the energy flux densities of the fields in (27-30):

$$
\begin{gathered}
\gamma m_{p} v=\frac{1}{c^{2}} \int\left(P_{x}+H_{x}+K_{x}+F_{x}\right) d V= \\
=\frac{4 \pi \gamma^{2} \gamma_{c}^{2} v}{9 c^{2}} \int \rho_{0}^{2}\left(\frac{\rho_{0 q}^{2}}{4 \pi \varepsilon_{0} \rho_{0}^{2}}-G+\eta+\sigma\right)\left(y^{2}+z^{2}\right) d V=0 . \\
0=\frac{1}{c^{2}} \int\left(P_{y}+H_{y}+K_{y}+F_{y}\right) d V= \\
=-\frac{4 \pi \gamma^{2} \gamma_{c}^{2} v}{9 c^{2}} \int \rho_{0}^{2}\left(\frac{\rho_{0 q}^{2}}{4 \pi \varepsilon_{0} \rho_{0}^{2}}-G+\eta+\sigma\right) y(x-v t) d V=0 . \\
0=\frac{1}{c^{2}} \int\left(P_{z}+H_{z}+K_{z}+F_{z}\right) d V= \\
=-\frac{4 \pi \gamma^{2} \gamma_{c}^{2} v}{9 c^{2}} \int \rho_{0}^{2}\left(\frac{\rho_{0 q}^{2}}{4 \pi \varepsilon_{0} \rho_{0}^{2}}-G+\eta+\sigma\right) z(x-v t) d V=0 .
\end{gathered}
$$

In (43), the mass $m_{p}$ is equal to zero due to relation (32), which is found in (43) as a separate multiplier within the integral. Zero on the left-hand side of (44) follows from the fact that the total momentum of the fields cannot be directed along the axes $O Y$ and $O Z$ of the reference frame $K_{L}$. Zero on the right-hand side of (44) is associated both with relation (32) and with the sphere's symmetry with respect to the limits of integration, as well as with the form of the expression under the integral sign. From (42-44) it follows that $m_{f}=m_{p}=0$. This means that the total mass-energies of the fields, found from the energy densities and energy flux densities inside the body, are equal to each other, because they are equal to zero.

Let us now consider the situation outside of the moving body, where there are only electromagnetic and gravitational fields. Using (38-39), similarly to (42), we find the total massenergy of the external fields with the help of the integral of the energy densities: 


$$
\begin{aligned}
& m_{f o}=\frac{1}{\gamma c^{2}} \int\left(W_{00}+U_{00}\right) d V= \\
& =\frac{\gamma \gamma_{c}^{2}}{8 \pi c^{2}}\left(\frac{q^{2}}{4 \pi \varepsilon_{0}}-G m^{2}\right) \int \frac{\left[(x-v t)^{2}+\left(y^{2}+z^{2}\right)\left(1+\frac{v^{2}}{c^{2}}\right)\right]}{\left[\gamma^{2}(x-v t)^{2}+y^{2}+z^{2}\right]^{3}} d V .
\end{aligned}
$$

Due to the motion of the body, the mass-energy of the external fields emerges, which is associated with the motion of these fields together with the body, that is, with the momenta of the fields. Similarly to (43-44), taking into account (36-37), for the body's motion along the axis $O X$ we obtain the following:

$$
\begin{gathered}
\gamma m_{p o} v=\frac{1}{c^{2}} \int\left(P_{x}+H_{x}\right) d V= \\
=\frac{\gamma^{2} \gamma_{c}^{2} v}{4 \pi c^{2}}\left(\frac{q^{2}}{4 \pi \varepsilon_{0}}-G m^{2}\right) \int \frac{y^{2}+z^{2}}{\left[\gamma^{2}(x-v t)^{2}+y^{2}+z^{2}\right]^{3}} d V . \\
\frac{1}{c^{2}} \int\left(P_{y}+H_{y}\right) d V=-\frac{\gamma^{2} \gamma_{c}^{2} v}{4 \pi c^{2}}\left(\frac{q^{2}}{4 \pi \varepsilon_{0}}-G m^{2}\right) \int \frac{y(x-v t)}{\left[\gamma^{2}(x-v t)^{2}+y^{2}+z^{2}\right]^{3}} d V=0 . \\
\frac{1}{c^{2}} \int\left(P_{z}+H_{z}\right) d V=-\frac{\gamma^{2} \gamma_{c}^{2} v}{4 \pi c^{2}}\left(\frac{q^{2}}{4 \pi \varepsilon_{0}}-G m^{2}\right) \int \frac{z(x-v t)}{\left[\gamma^{2}(x-v t)^{2}+y^{2}+z^{2}\right]^{3}} d V=0 .
\end{gathered}
$$

The integrals of the form (45-47) have been calculated many times in the works, devoted to $4 / 3$ problem, for example in $[15,16]$. Initially, the crux of the problem lay in the fact that the massenergy, associated with the momentum density of the electromagnetic field of the moving body, was about $4 / 3$ times greater than the mass-energy, associated with the energy density of the electromagnetic field of this body. In [14] it was shown that the $4 / 3$ problem also exists for the gravitational field of the moving body, specifically for the field both inside and outside the body. To calculate the integrals (45-47) it is convenient to introduce new coordinates as follows:

$$
x-v t=\frac{1}{\gamma} r \cos \theta, \quad y=r \sin \theta \cos \varphi, \quad z=r \sin \theta \sin \varphi .
$$

The volume element is given by the formula $d V=J d r d \theta d \varphi$, where $J$ is the determinant of the Jacobian matrix:

$$
J=\frac{\partial(x, y, z)}{\partial(r, \theta, \varphi)}=\left|\begin{array}{lll}
\frac{\partial x}{\partial r} & \frac{\partial x}{\partial \theta} & \frac{\partial x}{\partial \varphi} \\
\frac{\partial y}{\partial r} & \frac{\partial y}{\partial \theta} & \frac{\partial y}{\partial \varphi} \\
\frac{\partial z}{\partial r} & \frac{\partial z}{\partial \theta} & \frac{\partial z}{\partial \varphi}
\end{array}\right|
$$

After calculating this determinant we can see that $d V=\frac{1}{\gamma} r^{2} \sin \theta d r d \theta d \varphi$. 
Due to the Lorentz contraction during the motion along the axis $O X$, the sphere should take the form of a moving Heaviside ellipsoid, which has the following surface equation:

$$
\gamma^{2}(x-v t)^{2}+y^{2}+z^{2}=a^{2}
$$

From (48-49) it follows that the limits of integration over the volume in the new coordinates are as follows: the radius $r$ must vary from $a$ to $\infty$, and the angles $\theta$ and $\varphi$ vary in the same way as in spherical coordinates (from 0 up to $\pi$ for the angle $\theta$ and from 0 up to $2 \pi$ for the angle $\varphi$ ).

Substituting (48) and $d V$ in (45-46), we find:

$$
\begin{aligned}
& m_{f o}=\frac{\gamma_{c}^{2}}{8 \pi c^{2}}\left(\frac{q^{2}}{4 \pi \varepsilon_{0}}-G m^{2}\right) \int_{a}^{\infty} \frac{d r}{r^{2}} \int_{0}^{\pi}\left(1-\frac{v^{2}}{c^{2}}+\frac{2 v^{2}}{c^{2}} \sin ^{2} \theta\right) \sin \theta d \theta \int_{0}^{2 \pi} d \varphi= \\
& =\frac{\gamma_{c}^{2}}{2 a c^{2}}\left(\frac{q^{2}}{4 \pi \varepsilon_{0}}-G m^{2}\right)\left(1+\frac{v^{2}}{3 c^{2}}\right) . \\
& m_{p o}=\frac{\gamma_{c}^{2}}{4 \pi c^{2}}\left(\frac{q^{2}}{4 \pi \varepsilon_{0}}-G m^{2}\right) \int_{a}^{\infty} \frac{d r}{r^{2}} \int_{0}^{\pi} \sin ^{3} \theta d \theta \int_{0}^{2 \pi} d \varphi=\frac{2 \gamma_{c}^{2}}{3 a c^{2}}\left(\frac{q^{2}}{4 \pi \varepsilon_{0}}-G m^{2}\right) .
\end{aligned}
$$

If in (50) we neglect the factor $1+\frac{v^{2}}{3 c^{2}}$, then comparing it with (51) we obtain the well-known $4 / 3$ relation for the mass-energies of the external fields: $m_{p o} \approx \frac{4}{3} m_{f o}$. Thus, there is a necessity to explain the inequality of the field's mass-energies outside the moving body in case when these mass-energies are derived either from the field's energy flux density or from the field's energy density. As we can see, the inequality of mass energies holds separately both for the electromagnetic and gravitational fields, respectively.

In order to understand the $4 / 3$ problem we should pay attention to the following facts. In the stress-energy tensor of the electromagnetic field (7), the components included in equation (40) for the Poynting theorem outside the body, taking into account (21-22), equal:

$$
\begin{array}{cr}
W_{00}=\frac{\varepsilon_{0}}{2}\left[E^{2}+c^{2} B^{2}\right], & W_{01}=-\frac{P_{x}}{c}=-\varepsilon_{0} c[\mathbf{E} \times \mathbf{B}]_{x}, \\
W_{02}=-\frac{P_{y}}{c}=-\varepsilon_{0} c[\mathbf{E} \times \mathbf{B}]_{y}, & W_{03}=-\frac{P_{z}}{c}=-\varepsilon_{0} c[\mathbf{E} \times \mathbf{B}]_{z} .
\end{array}
$$

Similarly, in the stress-energy tensor of the gravitational field (9), the components included in equation (40) have the form:

$$
\begin{array}{cr}
U_{00}=-\frac{1}{8 \pi G}\left[\Gamma^{2}+c^{2} \Omega^{2}\right], & U_{01}=-\frac{H_{x}}{c}=\frac{c}{4 \pi G}[\boldsymbol{\Gamma} \times \mathbf{\Omega}]_{x}, \\
U_{02}=-\frac{H_{y}}{c}=\frac{c}{4 \pi G}[\boldsymbol{\Gamma} \times \mathbf{\Omega}]_{y}, & U_{03}=-\frac{H_{z}}{c}=\frac{c}{4 \pi G}[\boldsymbol{\Gamma} \times \mathbf{\Omega}]_{z} .
\end{array}
$$


If the components of the stress-energy tensor of the electromagnetic field (52) formed a fourvector of the following form: $W_{\mu}=\left(W_{00}, W_{01}, W_{02}, W_{03}\right)$, then after integration over the volume of these components we could reckon that the mass-energy derived from them would be the same both for the field energy and for the field momentum. An example is the four-momentum of the body, which contains the energy and the relativistic momentum, while both the energy and the momentum include the same invariant body mass.

However, the components (52) do not form a four-vector but are the components of the fourtensor. The same is true for the components (53) with respect to the gravitational field. Hence, it follows that we should not expect the equality of mass-energies of the form $m_{f o}=m_{p o}$, as we do not calculate these mass-energies from the components of four-vectors but from the components of four-tensors. Instead of the equality of mass-energies the calculations lead to the relation $m_{p o} \approx \frac{4}{3} m_{f o}$.

The difference between the mass-energies $m_{f o}$ and $m_{p o}$ follows from different mass-energy dependences in (50) and (51). But this very difference of the energy and momentum dependences of the electromagnetic and gravitational fields is required so that these fields outside the body would meet the generalized Poynting theorem (40). Besides, the Poynting theorem is the consequence of the equation of motion in a covariant form (1-2). At the same time, the expected mass-energy equality of the form $m_{f o}=m_{p o}$ is ungrounded. It could be expected for the case of the four-vectors, but not for the case of the four-tensors describing the fields. The $4 / 3$ problem in this case should be considered as an unexpected but logical paradox arising from the transition from four-vectors to four-tensors for describing the field. It also means that the stress-energy four-tensor of any field cannot be represented as a combination of a certain four-vector, composed of the time components of this tensor, and a three-dimensional stress tensor, composed of the space components of this tensor. This analogy is possible for the four-vector, consisting of the scalar and vector components, but it becomes inaccurate in case of tensors as more complex mathematical objects. The 4/3 coefficient in this case serves as a measure of this inaccuracy.

We should note that if we take a single field, for example, an electromagnetic or gravitational field, then the $4 / 3$ problem would take place for this field inside the body. However, if we simultaneously consider all the fields inside the body, including the pressure field and the acceleration field, as the general field components, then we would obtain the equality of massenergies: $m_{f}=m_{p}=0$, and in this case, the $4 / 3$ problem would disappear. This happens because all the fields compensate each other so that the invariant mass-energy of the general field vanishes. Besides, this compensation of fields is a consequence of the generalized Poynting theorem. We see that in an equilibrium system the compensation of motion of the particles of matter and field is possible due to the energy and momentum exchange between the matter and the field. However, where there is no matter, but there are only fundamental electromagnetic and gravitational fields, there is no compensation and we discover inequality of mass-energies, known as the $4 / 3$ problem.

\section{Relativistic Energy}

In [11] in the framework of the covariant theory of gravitation the relativistic energy of the physical system under consideration in the form of a fixed sphere with particles was calculated, taking into account the four fields, including the electromagnetic and gravitational fields, pressure field and acceleration field. The energy of the first two fields was taken into account not only inside, but also outside the sphere, since the electromagnetic and gravitational fields of the body extend to infinity. For the energy of the system we obtained the following expression: 


$$
\begin{aligned}
& E_{r}=m c^{2} \gamma_{c}^{2}-\frac{3 \eta m^{2} \gamma_{c}^{2}}{5 a}+\frac{3 q^{2} \gamma_{c}^{2}}{8 \pi \varepsilon_{0} a}-\frac{3 G m^{2} \gamma_{c}^{2}}{2 a}+m \wp_{c} \gamma_{c}-\frac{3 \sigma m^{2} \gamma_{c}^{2}}{10 a}- \\
& -\frac{3 q^{2} \gamma_{c}^{2}}{20 \pi \varepsilon_{0} a}+\frac{3 G m^{2} \gamma_{c}^{2}}{5 a}-\frac{\eta m^{2} \gamma_{c}^{2}}{10 a}-\frac{\sigma m^{2} \gamma_{c}^{2}}{10 a}
\end{aligned}
$$

When calculating the energy of the particles, that are in the gravitational potential $\psi_{i}$ inside the sphere and have the Lorentz factor $\gamma^{\prime}$, in [11] we used the formula:

$$
\begin{aligned}
& \int \rho_{0} \psi_{i} \gamma^{\prime} d x^{1} d x^{2} d x^{3}= \\
& =\frac{G \rho_{0} c^{3} \gamma_{c}^{2}}{\eta \sqrt{4 \pi \eta \rho_{0}}} \int\left(\begin{array}{l}
\cos \left(\frac{a}{c} \sqrt{4 \pi \eta \rho_{0}}\right)- \\
-\frac{c}{r \sqrt{4 \pi \eta \rho_{0}}} \sin \left(\frac{r}{c} \sqrt{4 \pi \eta \rho_{0}}\right)
\end{array}\right) \frac{1}{r} \sin \left(\frac{r}{c} \sqrt{4 \pi \eta \rho_{0}}\right) r^{2} \sin \theta d r d \theta d \phi= \\
& =\frac{G c^{4} \gamma_{c}^{2}}{\eta^{2}}\left[\cos \left(\frac{a}{c} \sqrt{4 \pi \eta \rho_{0}}\right)\left(\begin{array}{l}
\frac{c}{\sqrt{4 \pi \eta \rho_{0}}} \sin \left(\frac{a}{c} \sqrt{4 \pi \eta \rho_{0}}\right)- \\
-a \cos \left(\frac{a}{c} \sqrt{4 \pi \eta \rho_{0}}\right)
\end{array}\right)-\frac{a}{2}+\frac{c}{4 \sqrt{4 \pi \eta \rho_{0}}} \sin \left(\frac{2 a}{c} \sqrt{4 \pi \eta \rho_{0}}\right)\right] \approx \\
& \approx-\frac{3 G m^{2} \gamma_{c}^{2}}{2 a} \text {. }
\end{aligned}
$$

The energy value $-\frac{3 G m^{2} \gamma_{c}^{2}}{2 a}$ was obtained based on the fact that the periodic functions such as $\cos \left(\frac{a}{c} \sqrt{4 \pi \eta \rho_{0}}\right)$ and $\sin \left(\frac{a}{c} \sqrt{4 \pi \eta \rho_{0}}\right)$ in the square brackets had been expanded into the second-order terms. Now we wish to increase the accuracy of calculations and to expand the periodic functions into the third-order or even fourth-order terms. In particular, it gives the following:

$$
\int \rho_{0} \psi_{i} \gamma^{\prime} d x^{1} d x^{2} d x^{3} \approx-\frac{6 G m^{2} \gamma_{c}^{2}}{5 a}\left(1-\frac{4 \eta m}{7 a c^{2}}\right)
$$

The same is obtained for the energy of the charged particles in the electrical potential $\varphi_{i}$ inside the sphere - the total energy of these particles decreases from the value $\frac{3 q^{2} \gamma_{c}^{2}}{8 \pi \varepsilon_{0} a}$ to the value $\frac{3 q^{2} \gamma_{c}^{2}}{10 \pi \varepsilon_{0} a}\left(1-\frac{4 \eta m}{7 a c^{2}}\right)$. This means that the energy of the system changes and becomes equal to the following expression:

$$
\begin{aligned}
& E_{r}=m c^{2} \gamma_{c}^{2}-\frac{3 \eta m^{2} \gamma_{c}^{2}}{5 a}+\frac{3 q^{2} \gamma_{c}^{2}}{10 \pi \varepsilon_{0} a}-\frac{6 G m^{2} \gamma_{c}^{2}}{5 a}+m \wp_{c} \gamma_{c}-\frac{3 \sigma m^{2} \gamma_{c}^{2}}{10 a}- \\
& -\frac{3 q^{2} \gamma_{c}^{2}}{20 \pi \varepsilon_{0} a}+\frac{3 G m^{2} \gamma_{c}^{2}}{5 a}-\frac{\eta m^{2} \gamma_{c}^{2}}{10 a}-\frac{\sigma m^{2} \gamma_{c}^{2}}{10 a} .
\end{aligned}
$$


In (54) the mass $m$ is the product of the mass density $\rho_{0}$ by the volume of the sphere with the particles, and the charge $q$ is defined similarly as the product of the charge density $\rho_{0 q}$ by the volume of the sphere. However, outside the sphere the potentials of the electromagnetic and gravitational fields are determined with the help of the gravitational mass $m_{b}$ and the electric charge $q_{b}$. For these masses we obtain the following [11]:

$$
m \gamma_{c} \approx m_{b}+\frac{3 \eta m_{b}^{2}}{10 a c^{2} \gamma_{c}}, \quad q \gamma_{c} \approx q_{b}+\frac{3 \eta m_{b} q_{b}}{10 a c^{2} \gamma_{c}}
$$

The Lorentz factor for the particles in the center of the sphere and the scalar potential of the pressure field in the center of the sphere are expressed in terms of the corresponding quantities on the surface of the sphere:

$$
\gamma_{c} \approx \gamma_{s}+\frac{\eta m_{b}}{2 a c^{2}}, \quad \wp_{c} \approx \wp_{s}+\frac{\sigma m_{b}}{2 a}
$$

Substituting the sum $\eta+\sigma$ from (32) into (54), taking into account (55) and (56), for the energy and the invariant (inertial) mass of the system $M$ we find the following:

$$
E_{r}=M c^{2}=m_{b} c^{2} \gamma_{s}-\frac{G m_{b}^{2}}{2 a}+\frac{q_{b}^{2}}{8 \pi \varepsilon_{0} a}+m_{b} \wp_{s} .
$$

For the majority of cosmic bodies we can assume that for the body's surface the Lorentz factor is $\gamma_{s} \approx 1$ and the scalar potential of the pressure field is $\wp_{s} \approx 0$.

The formula for the energy (57) differs from the formula for the energy of the system in [11], which has the following form:

$$
E_{r}=M c^{2}=m_{b} c^{2} \gamma_{s}-\frac{3 G m_{b}^{2}}{10 a}+\frac{3 q_{b}^{2}}{40 \pi \varepsilon_{0} a}+m_{b} \wp_{s} .
$$

The difference between the formulas for the energy, excluding specification of the energy of particles in the potentials of the gravitational and electromagnetic fields in (54), also arises from the fact that in [11] we used the relations for the fields' coefficients in accordance with the formula: $\eta=\sigma=3 G-\frac{3 q^{2}}{4 \pi \varepsilon_{0} m^{2}}$. In turn, these relations have been determined in [12] as a result of integrating the equation of motion (1) with respect to the infinite four-dimensional volume. The subsequent application of the four-dimensional divergence theorem with transition from integrating the divergence of the sum of the stress-energy tensors of the four fields with respect to the fourdimensional volume to integrating the sum of these tensors with respect to the three-dimensional volume gives the integral four-vector:

$$
\mathbb{Q}^{\alpha}=\int \tau^{\alpha 0} d S_{0}=\int\left(W^{\alpha 0}+U^{\alpha 0}+B^{\alpha 0}+P^{\alpha 0}\right) \sqrt{-g} d x^{1} d x^{2} d x^{3}
$$

In order to always reserve the four-vector $\mathbb{Q}^{\alpha}$ it was equated to zero. As a result, for the field coefficients we obtained the formula: $\eta=\sigma=3 G-\frac{3 q^{2}}{4 \pi \varepsilon_{0} m^{2}}$. Now we see that this formula is only a 
first approximation to the truth. In fact, during integration in (58) we took into account the contribution of the electromagnetic and gravitational fields both inside and outside the moving sphere. However, the generalized Poynting theorem considers the energy and fluxes of the fields locally, in any small volume of space. There are at least four fields inside the body, and outside the body there are only two fields, because here there are no pressure field and acceleration field of the matter particles. Consequently, the fields' content in local volumes inside and outside the body is different, and the four-vector $\mathbb{Q}^{\alpha}$ must be calculated separately for the internal body volume and for the volume outside the body.

On the other hand, (58) contains the time components of the stress-energy tensors, and we have shown in the previous section that these components do not form any four-vector. This leads to the fact that $\mathbb{Q}^{\alpha}$ is equal to zero only inside the body, as it follows from the generalized Poynting theorem in section 3.1. As for the space outside the body, $\mathbb{Q}^{\alpha}$ here is not equal to zero. Thus, the concept of the integral four-vector in the form of (58) is applicable only inside the cosmic bodies or for infinitely large systems of matter and fields, when the fundamental fields outside the system do not make a significant contribution into the calculations of energy and momentum.

In addition, (58) is not a complete expression, since during derivation of (58) in [12] we did not take into account the field energy fluxes at the boundaries of the infinite volume.

More precise application of the divergence theorem to (1) results for the Cartesian coordinates of the Lorentzian reference frame, in which the Christoffel symbols $\Gamma_{\beta \lambda}^{\alpha}$ are equal to zero, in the following expression for the four fields:

$$
\begin{aligned}
& \mathbb{Q}^{\alpha}=\int \nabla_{\beta} \tau^{\alpha \beta} \sqrt{-g} d x^{0} d x^{1} d x^{2} d x^{3}= \\
& =\int \tau^{\alpha \beta} d S_{\beta}=\int\left(W^{\alpha 0}+U^{\alpha 0}+B^{\alpha 0}+P^{\alpha 0}\right) \sqrt{-g} d x^{1} d x^{2} d x^{3}+ \\
& +\int\left(W^{\alpha 1}+U^{\alpha 1}+B^{\alpha 1}+P^{\alpha 1}\right) \sqrt{-g} d x^{0} d x^{2} d x^{3}+\int\left(W^{\alpha 2}+U^{\alpha 2}+B^{\alpha 2}+P^{\alpha 2}\right) \sqrt{-g} d x^{0} d x^{1} d x^{3}+ \\
& +\int\left(W^{\alpha 3}+U^{\alpha 3}+B^{\alpha 3}+P^{\alpha 3}\right) \sqrt{-g} d x^{0} d x^{1} d x^{2} .
\end{aligned}
$$

In this expression $d x^{0}=c d t$, and $d S_{\beta}$ is a four-vector, composed of the corresponding products of the coordinate differentials; this four-vector is dual to the three-dimensional hypersurface, which surrounds the four-dimensional volume. In the calculation of $\mathbb{Q}^{\alpha}$ we need to calculate four integrals, in each of which the integrands must be expressed with the help of the hypersurface equation in terms of those coordinates, by which the integration is performed. In this form $\mathbb{Q}^{\alpha}$ is reserved as some four-dimensional quantity, since the expression for such $\mathbb{Q}^{\alpha}$ represents an integral expression of the generalized Poynting theorem (compare with (19-20)).

Conservation of $\mathbb{Q}^{\alpha}$ in each closed physical system means that such a physical system has not only some constant four-momentum, but also has unchanged distribution of the energies of the fields and their fluxes. However, the existence of the $4 / 3$ problem means that the quantity $\mathbb{Q}^{\alpha}$ is not a four-vector and does not determine the four-momentum of the system, as it is assumed in the general theory of relativity. The difference between the four-momentum and $\mathbb{Q}^{\alpha}$ follows from the fact that the first refers to the total energy and momentum of all the particles of the system's matter, and the second refers to the energy and energy fluxes (momentum fluxes) of all the system's fields.

\section{Conclusion}

The analysis of the field equations for a generally non-rotating physical system of particles and fields in the form of a sphere shows that with the help of the generalized Poynting theorem in the framework of the covariant theory of gravitation it is possible to understand some regularities that have no simple explanation in the standard approach. All the fields acting in the system 
collectively form the general field. We distinguish two main components of the general field: the charge component of the general field is represented by the electromagnetic field, and the mass component of the general field consists of the gravitational field, acceleration field, pressure field, dissipation field and the macroscopic fields of strong and weak interactions. Being limited only by the four basic fields, in (15) we express the coefficient $\varpi$ of the mass component of the general field in terms of the acceleration field coefficient $\eta$, the pressure field coefficient $\sigma$ and the gravitational constant $G$. Next we show that if in the stationary system we consider all the fields independent of each other, then inside the body the stress-energy tensor of the general field's mass component is equal to the sum of the stress-energy tensors of all the fields, except for the electromagnetic field.

In (32) we find correlation between the fields' coefficients $\eta, \sigma, G$ and the vacuum permittivity $\varepsilon_{0}$, which exists inside the bodies in equilibrium state. This allows us to solve the $4 / 3$ problem, which has not had exact solution for a long time. Firstly, the $4 / 3$ problem exists for each field, both inside and outside the body. However, inside the body the combination of fields is such that they compensate each other and for the general field the $4 / 3$ problem disappears. Secondly, the explanation of the $4 / 3$ problem outside of the body for the electromagnetic and gravitational fields consists in the fact that the field energy density and the field energy flux vector, which are part of the time components of the stress-energy tensor, do not form a four-vector. This does not allow these components to have the same mass-energies for the moving body, which represents the $4 / 3$ problem.

In (54) we represent the relativistic energy of the system of particles and fields under consideration. The energy consists of two parts, the first of which is the sum of the energies of particles under the influence of the fields. The second part is associated with the sum of the field energies as such. If in [11] the second part of the energy, associated with the fields, was equated to zero, then in (54) it was no longer the case. This was due to the more precise correlation between the field coefficients in (32), which differed from what was used in [11]. In (57) we express the relativistic energy in terms of the mass $m_{b}$ and the charge of the system $q_{b}$.

The generalized Poynting theorem for the four fields is presented in (20). If we take the integral over the volume of all the terms in (20) and replace the volume integral of the divergence of the sum of vectors by the integral of this sum of vectors over a closed surface, surrounding the volume, we obtain the following:

$$
\frac{\partial}{\partial t} \int_{V}\left(W_{00}+U_{00}+B_{00}+P_{00}\right) d V=-\int_{S}(\mathbf{P}+\mathbf{H}+\mathbf{K}+\mathbf{F}) \cdot \mathbf{n} d S
$$

where $\mathbf{n}$ denotes the normal vector to the surface.

In this relation the rate of change in the energy of the fields in an arbitrary volume is exactly compensated by the fluxes of the fields' energy inside this volume through its surface. From the standpoint of the information theory, the entire information describing the fluxes of particles and field quanta moving through the surface, does not disappear and is summed up in the information describing the particles and field quanta inside the volume. This reminds of the holographic principle in the string theory, which suggests that the quantum information, associated with the particles and fields that got into the black hole, is reflected in the fluctuations of the visible gravitational horizon. But in contrast to the holographic principle, the generalized Poynting theorem is more fundamental, as it is substantiated by the entire apparatus of the field theory.

A peculiarity of our approach is the fact that the matter is fully described in the concept of field, so that instead of the stress-energy tensor of matter we use the traceless symmetric stressenergy tensor of the acceleration field. The stress-energy tensors of the electromagnetic and gravitational fields, the pressure field and other fields, found by a standard procedure [6], are also traceless. This property of fields is important both in writing the equation for the metric in the covariant theory of gravitation and in gauge of the relativistic energy of the system. As a result, it 
leads to uniformity of dependences of potentials and field strengths inside the body [17] and to the possibility of using the generalized Poynting theorem for simple description of the properties of systems of particles and fields.

The relation for the field coefficients (32) was derived from the equation of motion (4) separately for the time and space components of the tensors. In the first case, this led to the generalized Poynting theorem for densities and energy fluxes, and in the second case this led to the equation of the particles' motion under the action of forces. Besides, in order to derive (32) we needed to take into account the particular form of the field tensors. We will now show that relation (32) for the relativistic uniform system can be obtained even without calculating the field tensors.

Let us multiply (8) by the ratio of densities $\frac{\rho_{0 q}}{\rho_{0}}$, assuming this ratio to be constant, and then add up with (12-14), taking into account that $J^{\alpha}=\rho_{0} u^{\alpha}, j^{\alpha}=\rho_{0 q} u^{\alpha}$ :

$$
\nabla_{\beta}\left(\Phi^{\alpha \beta}+\frac{\rho_{0 q}}{\rho_{0}} F^{\alpha \beta}+u^{\alpha \beta}+f^{\alpha \beta}\right)=\frac{4 \pi}{c^{2}}\left(G-\frac{\rho_{0 q}^{2}}{4 \pi \varepsilon_{0} \rho_{0}^{2}}-\eta-\sigma\right) J^{\alpha} .
$$

Equation (4) can be written as follows:

$$
\left(\Phi^{\alpha \beta}+\frac{\rho_{0 q}}{\rho_{0}} F^{\alpha \beta}+u^{\alpha \beta}+f^{\alpha \beta}\right) J_{\beta}=0,
$$

We will now use the equation for the metric in the covariant theory of gravitation and the continuity relations arising from the field equations [5]:

$$
\begin{gathered}
R_{\alpha \beta}-\frac{1}{4} R g_{\alpha \beta}=-\frac{1}{2 c k}\left(U_{\alpha \beta}+W_{\alpha \beta}+B_{\alpha \beta}+P_{\alpha \beta}\right), \\
R_{\alpha \beta} \Phi^{\alpha \beta}=-\frac{4 \pi G}{c^{2}} \nabla_{\alpha} J^{\alpha}, \\
R_{\alpha \beta} u^{\alpha \beta}=\frac{4 \pi \eta}{c^{2}} \nabla_{\alpha} J^{\alpha}, \quad R_{\alpha \beta} F^{\alpha \beta}=\frac{1}{\varepsilon_{0} c^{2}} \nabla_{\alpha} j^{\alpha}, \\
R_{\alpha \beta} f^{\alpha \beta}=\frac{4 \pi \sigma}{c^{2}} \nabla_{\alpha} J^{\alpha} .
\end{gathered}
$$

The gravitational field tensor is determined using the four-potential $D^{\beta}$ as follows: $\Phi^{\alpha \beta}=\nabla^{\alpha} D^{\beta}-\nabla^{\beta} D^{\alpha}$. Hence it follows that $g_{\alpha \beta} \Phi^{\alpha \beta}=\nabla_{\beta} D^{\beta}-\nabla_{\alpha} D^{\alpha}=0$, and the same should hold for the tensors of other fields. Let us now multiply one after another the tensors of each field by the both sides of equation (61), while for the electromagnetic field we should multiply (61) by $\frac{\rho_{0 q}}{\rho_{0}} F^{\alpha \beta}$, and then sum up everything:

$$
R_{\alpha \beta}\left(\Phi^{\alpha \beta}+\frac{\rho_{0 q}}{\rho_{0}} F^{\alpha \beta}+u^{\alpha \beta}+f^{\alpha \beta}\right)=-\frac{1}{2 c k}\left(U_{\alpha \beta}+W_{\alpha \beta}+B_{\alpha \beta}+P_{\alpha \beta}\right)\left(\Phi^{\alpha \beta}+\frac{\rho_{0 q}}{\rho_{0}} F^{\alpha \beta}+u^{\alpha \beta}+f^{\alpha \beta}\right) .
$$

Let us take into account relations (62) on the left-hand side of this equation:

$$
-\frac{4 \pi}{c^{2}}\left(G-\frac{\rho_{0 q}^{2}}{4 \pi \varepsilon_{0} \rho_{0}^{2}}-\eta-\sigma\right) \nabla_{\alpha} J^{\alpha}=-\frac{1}{2 c k}\left(U_{\alpha \beta}+W_{\alpha \beta}+B_{\alpha \beta}+P_{\alpha \beta}\right)\left(\Phi^{\alpha \beta}+\frac{\rho_{0 q}}{\rho_{0}} F^{\alpha \beta}+u^{\alpha \beta}+f^{\alpha \beta}\right) .(63
$$


The field tensors in the equation of motion (60) arise from the contributions of all the system's particles and act on the four-current $J_{\beta}$, which is a local quantity. Consequently, the sum of the field tensors in (60) does not depend on $J_{\beta}$ and must be equal to zero:

$$
\Phi^{\alpha \beta}+\frac{\rho_{0 q}}{\rho_{0}} F^{\alpha \beta}+u^{\alpha \beta}+f^{\alpha \beta}=0 .
$$

Then the equality for the field coefficients (32) follows from (59) and (63). On the other hand, we can take the covariant derivative $\nabla_{\alpha}$ in (59) and substitute the result into (63):

$-\nabla_{\alpha} \nabla_{\beta}\left(\Phi^{\alpha \beta}+\frac{\rho_{0 q}}{\rho_{0}} F^{\alpha \beta}+u^{\alpha \beta}+f^{\alpha \beta}\right)=-\frac{1}{2 c k}\left(U_{\alpha \beta}+W_{\alpha \beta}+B_{\alpha \beta}+P_{\alpha \beta}\right)\left(\Phi^{\alpha \beta}+\frac{\rho_{0 q}}{\rho_{0}} F^{\alpha \beta}+u^{\alpha \beta}+f^{\alpha \beta}\right)$.

The simplest solution of this equation for the field tensors is relation (64). In addition, it can be noted that in the system under consideration with the uniform mass and charge density, the sum of the stress-energy tensors of all the fields inside the body also becomes equal to zero. For the time components of the tensors, this can be seen from (31) and (32). The stress-energy tensors of all the fields in (7), (9-11) have the same form. If (17) and (32) are valid, then the sum of the stress-energy tensors of all the fields becomes equal to zero

$$
U_{\alpha \beta}+W_{\alpha \beta}+B_{\alpha \beta}+P_{\alpha \beta}=\frac{c^{2}}{4 \pi \eta^{2}} g^{\alpha \kappa} u_{\alpha \beta} u_{\kappa \sigma}\left(-\delta_{\mu}^{\beta} \delta_{v}^{\sigma}+\frac{1}{4} g_{\mu \nu} g^{\sigma \beta}\right)\left(-G+\frac{\rho_{0 q}^{2}}{4 \pi \varepsilon_{0} \rho_{0}^{2}}+\eta+\sigma\right)=0 \text {. }
$$

and the equation for the metric (61) inside the body acquires a very simple form:

$$
R_{\alpha \beta}-\frac{1}{4} R g_{\alpha \beta}=0
$$

\section{References}

[1]Poynting J. H. On the Transfer of Energy in the Electromagnetic Field. Philosophical Transactions of the Royal Society of London, Vol. 175, pp. 343-361 (1884). doi:10.1098/rstl.1884.0016.

[2]Richter, E.; Florian, M.; Henneberger, K. Poynting's theorem and energy conservation in the propagation of light in bounded media. Europhysics Letters, Vol. 81 (6), pp. 67005 (2008). doi:10.1209\%2F0295-5075\%2F81\%2F67005.

[3]Fedosin S.G. The Concept of the General Force Vector Field. OALib Journal, Vol. 3, pp. 1-15 (2016), e2459. doi:10.4236/oalib.1102459.

[4]Fedosin S.G. Two components of the macroscopic general field. Reports in Advances of Physical Sciences, Vol. 1, No. 2, 1750002, 9 pages (2017). doi:10.1142/S2424942417500025.

[5]Fedosin S.G. About the cosmological constant, acceleration field, pressure field and energy. Jordan Journal of Physics, Vol. 9, No. 1, pp. 1-30 (2016). doi:10.5281/zenodo.889304.

[6]Fedosin S.G. The procedure of finding the stress-energy tensor and vector field equations of any form. Advanced Studies in Theoretical Physics, Vol. 8, pp. 771-779 (2014). doi:10.12988/astp.2014.47101.

[7]Jefimenko O. (2000). Causality, Electromagnetic Induction and Gravitation. Star City, Electret Scientific Company. 
[8]Fock V. A. (1964). The Theory of Space, Time and Gravitation. Macmillan.

[9]Fedosin S.G. Four-Dimensional Equation of Motion for Viscous Compressible and Charged Fluid with Regard to the Acceleration Field, Pressure Field and Dissipation Field. International Journal of Thermodynamics, Vol. 18, pp. 13-24 (2015). doi:10.5541/ijot.5000034003.

[10]Fedosin S. G. Fizika i filosofiia podobiia ot preonov do metagalaktik. (Perm, 1999). ISBN 58131-0012-1.

[11]Fedosin S.G. Relativistic Energy and Mass in the Weak Field Limit. Jordan Journal of Physics, Vol. 8, No. 1, pp. 1-16 (2015). doi:10.5281/zenodo.889210.

[12]Fedosin S.G. The Integral Energy-Momentum 4-Vector and Analysis of 4/3 Problem Based on the Pressure Field and Acceleration Field. American Journal of Modern Physics, Vol. 3, No. 4, pp. 152-167 (2014). doi: 10.11648/j.ajmp.20140304.12.

[13]Fedosin S.G. The virial theorem and the kinetic energy of particles of a macroscopic system in the general field concept. Continuum Mechanics and Thermodynamics, Vol. 29, Issue 2, pp. 361-371 (2017). doi:10.1007/s00161-016-0536-8.

[14]Fedosin S.G. 4/3 Problem for the Gravitational Field. Advances in Physics Theories and Applications, Vol. 23, pp. 19-25 (2013). doi:10.5281/zenodo.889383.

[15]Heaviside, Oliver. Electromagnetic waves, the propagation of potential, and the electromagnetic effects of a moving charge. Electrical papers, 2, pp. 490-499 (1888/1894). doi:10.1017/CBO9780511983139.017.

[16] Searle G.F.C. On the steady motion of an electrified ellipsoid. The Philosophical Magazine Series 5, Vol. 44 (269), pp. 329-341(1897). doi:10.1088/1478-7814/15/1/323.

[17]Fedosin S.G. Estimation of the physical parameters of planets and stars in the gravitational equilibrium model. Canadian Journal of Physics, Vol. 94, No. 4, pp. 370-379 (2016). doi:10.1139/cjp-2015-0593. 